\title{
The multi-protein family of sulfotransferases in plants: composition, occurrence, substrate specificity, and functions
}

\section{Felix Hirschmann, Florian Krause and Jutta Papenbrock *}

Institute of Botany, Leibniz University Hannover, Hannover, Germany

\section{Edited by:}

Stanislav Kopriva, University of

Cologne, Germany

\section{Reviewed by:}

Masami Yokota Hirai, RIKEN Plant

Science Center, Japan

Tamara Gigolashvili, University of

Cologne - Biocenter, Germany

Frédéric Marsolais, Agriculture and

Agri-Food Canada, Canada

\section{${ }^{*}$ Correspondence:}

Jutta Papenbrock, Institute of Botany, Leibniz University Hannover,

Herrenhäuser Straße 2, D-30419

Hannover, Germany

e-mail: jutta.papenbrock@botanik.

uni-hannover.de
All members of the sulfotransferase (SOT, EC 2.8.2.-) protein family transfer a sulfuryl group from the donor $3^{\prime}$-phosphoadenosine $5^{\prime}$-phosphosulfate (PAPS) to an appropriate hydroxyl group of several classes of substrates. The primary structure of these enzymes is characterized by a histidine residue in the active site, defined PAPS binding sites and a longer SOT domain. Proteins with this SOT domain occur in all organisms from all three domains, usually as a multi-protein family. Arabidopsis thaliana SOTs, the best characterized SOT multi-protein family, contains 21 members. The substrates for several plant enzymes have already been identified, such as glucosinolates, brassinosteroids, jasmonates, flavonoids, and salicylic acid. Much information has been gathered on desulfoglucosinolate (dsGl) SOTs in A. thaliana. The three cytosolic dsGI SOTs show slightly different expression patterns. The recombinant proteins reveal differences in their affinity to indolic and aliphatic dsGls. Also the respective recombinant dsGI SOTs from different $A$. thaliana ecotypes differ in their kinetic properties. However, determinants of substrate specificity and the exact reaction mechanism still need to be clarified. Probably, the threedimensional structures of more plant proteins need to be solved to analyze the mode of action and the responsible amino acids for substrate binding. In addition to $A$. thaliana, more plant species from several families need to be investigated to fully elucidate the diversity of sulfated molecules and the way of biosynthesis catalyzed by SOT enzymes.

Keywords: Arabidopsis thaliana,

glucosinolate, histidine residue, phosphoadenosine $5^{\prime}$-phosphosulfate, sulfotransferase

\section{INTRODUCTION}

Members of the sulfotransferase (SOT) family have been found in all organisms investigated to date. All of these enzymes catalyze the transfer of a sulfuryl group from $3^{\prime}$-phosphoadenosine $5^{\prime}$-phosphosulfate (PAPS) to an appropriate hydroxyl group (Figure 1), hydroxyl amine or unprotonated amine of various substrates with the parallel formation of PAP.

The SOTs catalyze the sulfation of a wide range of compounds and produce sulfate esters, sulfamates, and sulfate conjugates (Klaassen and Boles, 1997). A sulfate conjugate is more water soluble than a non-sulfated molecule (Weinshilboum and Otterness, 1994), thus facilitating excretion and bioactivation.

Due to the unifying use of the co-substrate PAPS, all SOT proteins are characterized by a histidine residue in the active site, defined PAPS binding sites and a defined SOT domain (Pfam: PF00685; Finn etal., 2014). Proteins with this SOT domain occur in all organisms from all three domains investigated so far, usually as a multi-protein family. Originally, the SOT proteins in mammals were classified on the basis of their affinity for different classes of substrates. One group of SOT proteins, mainly membrane-associated, accepts as substrates macromolecules, such as proteins and peptides, and glycosaminoglycans (Niehrs etal., 1994). The second group, usually soluble proteins, accepts as substrates small organic molecules, such as flavonoids, steroids, and xenobiotics, with diverse chemical structures. In plants, the best criteria for forming subgroups within the multi-protein family is still a matter of debate, because either sequence identity/similarity or their substrate specificity could be chosen. Several compounds have been found in different plant species, such as: brassinosteroids, coumarins, flavonoids, gibberellic acids, glucosinolates (Gls), phenolic acids, sulfate esters such as choline-O-sulfate, and terpenoids that might be sulfated by SOT proteins. However, only for some of these substrates has the catalyzing SOT protein been identified. Not all sulfated compounds are necessarily sulfated by SOTs. Sulfolipids contain a 6-deoxy-6-sulfoglucose sugar head group, referred to as sulfoquinovose. The sulfoquinovose precursor UDP-sulfoquinovose is biosynthesized from UDP-glucose by a UDP-sulfoquinovose synthase associated with a ferredoxin-dependent glutamate synthase using sulfite as cosubstrate (Shimojima et al., 2005). Much information has been gathered on desulfo-glucosinolate (dsGl) SOTs in Arabidopsis, differing in their affinity to indolic and aliphatic dsGls. However, determinants of substrate specificity and the exact reaction mechanism still need to be clarified. Probably, the three-dimensional structures of more plant proteins have to be solved to analyze the mode of action and the responsible amino acids for substrate binding. 


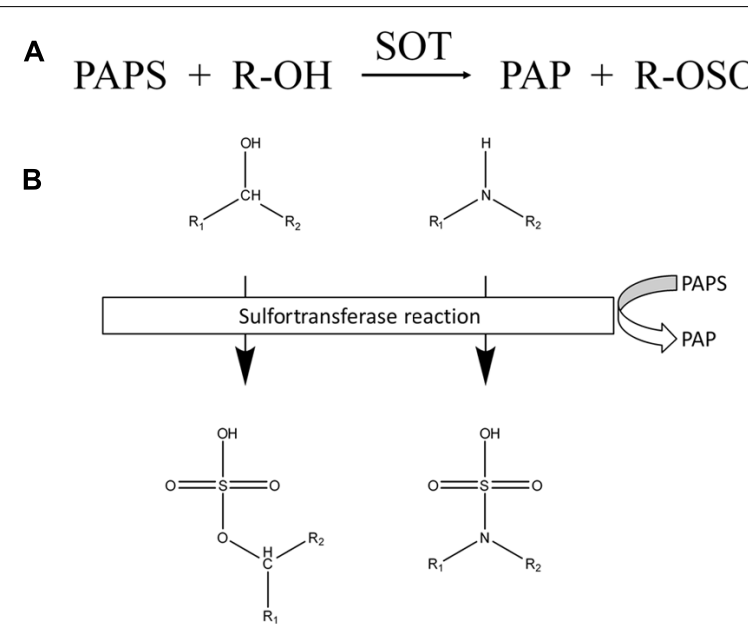

FIGURE 1 | Reactions catalyzed by SOTs. (A) Chemical equation of reactions catalyzed by SOTs. (B) Schematic overview of SOT targets. Chemical structure of targeted hydroxyl and amide groups and their sulfated products, sulfate ester and the sulfamate group.

\section{PRIMARY STRUCTURE OF SOTS, PAPS BINDING REGIONS, AND ALIGNMENT OF THE HIGHLY CONSERVED REGIONS}

Generally, SOTs can be divided into membrane-bound proteins and soluble cytosolic proteins. So far, only a few membranebound SOTs have been characterized in plants. They are either bound to the plasma membrane, as shown for the gallic acid glucoside SOT from Mimosa pudica L. (Varin et al., 1997a), or localized in the Golgi apparatus, as shown for the tyrosylprotein SOTs (TPSTs) from Asparagus officinalis L. (Hanai et al., 2000) and Arabidopsis thaliana (L.) Heynh. (Komori et al., 2009). The term cytosolic SOT might indicate a localization in the cytoplasm, yet the name implies that the proteins can be purified from plant cells and kept in solution (Hernàndez-Sebastiá et al., 2008). The exact localization of most cytosolic plant SOTs still remain unknown.

Sequence alignments of eleven cytosolic SOTs from plants, animals, and bacteria resulted in the identification of four highly conserved regions I to IV (Marsolais and Varin, 1995; Figure 2). Further analyses showed that especially the regions I and IV are highly conserved, for example throughout the SOT family of A. thaliana (Klein and Papenbrock, 2004). The regions I, II, and IV are responsible for the binding of the co-substrate PAPS (Varin et al., 1997b). The first structural approach to clarify the relevance of the regions for PAPS binding was determined by Xray crystallography analyses of a mouse estrogen SOT (Kakuta et al., 1997). Region I is localized close to the N-terminus and includes the PAPS binding domain (PSB domain) that interacts with the $5^{\prime}$-phosphate of PAPS. Region II starts with a characteristic highly conserved histidine, responsible for proton acceptance during the sulfuryl transfer (Kakuta et al., 1998). In the C-terminal part of region II the two amino acids Arg130 and Ser 138 are responsible for the binding of the $3^{\prime}$-phosphate of PAP and form a $3^{\prime}$ P-motif (Kakuta et al., 1997). This motif can be found in 18 SOTs out of 22 from A. thaliana and from almost all other plant SOTs. In many plant SOTs, a conserved hydrophilic site containing poly-glutamic acid (Poly-Glu) of unknown function can be found between region III and IV. Similar motifs have been found in a human chondroitin 6-SOT, but at a different position (Fukuta et al., 1998). Region IV is localized at the C-terminus and contains a P-loop related GxxGxxK motif (Figure 2).

In A. thaliana, 18 protein sequences with high similarity to known SOTs have been originally identified by BLAST approaches (Klein and Papenbrock, 2004). Later, another three SOTs were added (Klein and Papenbrock, 2008). These were formerly annotated in NCBI as "nodulation-related protein" and are now annotated as "P-loop containing triphosphate hydrolase family protein." In addition to these SOTs, a TPST has been identified (Komori et al., 2009). Furthermore, a not yet literarily mentioned protein, Q9SCR3, with a Sulfotransfer_l domain (PF00685), is available in the Pfam database [http://pfam.xfam.org/protein/Q9SCR3 (accessed 23.06.2014)]. About $75 \%$ of the amino acid sequence is identical to A. thaliana SOT (AtSOT19). Therefore, it might be a redundant entry or a product of a different splicing process. Interestingly, AtTPST is exceptional in its structure compared to the remaining AtSOTs. With 500 amino acids, it is not only bigger, but is also the solely identified transmembrane, cis-Golgi localized AtSOT. Furthermore, it shows no sequence similarity to human TPSTs; and no other typical features like the regions I to IV and the characteristic highly conserved histidine were identified (Komori et al., 2009). It is also the only Arabidopsis SOT that contains a Sulfotransfer_2 domain (PF03567), instead of a Sulfotranfer_1 domain (PF00685). Hence, it is only associated by function and not by sequence. Excluding the pseudogenic sequence AtSOT2 and TPST, the SOT protein lengths range between 273 and 403 amino acids with an average length of 321 amino acids. Only seven out of 21 AtSOTs contain introns.

There are several nomenclatures for $A$. thaliana SOTs used in the literature. The most common ones are listed in Table 1, including information about the preferred SOT substrates. In this review, the nomenclature first introduced by Klein and Papenbrock (2004) is used.

So far, only one SOT from plants (AtSOT12 from A. thaliana) was structurally solved (Smith etal., 2004). Therefore, most SOT proteins lack structural analyses and detailed enzymological characterizations. The identified motifs only give a hint on the proteins' general function as a SOT, but no information about their specificity and affinity toward certain substrates.

Most proteins identified as putative SOTs contain at least one out of seven related Pfam motifs that are based on Hidden Markov Models (HMM). The most important HMMs referring to SOTs are the SOT domains Sulfotransfer_1 (PF00685), Sulfotransfer_2 (PF03567), and Sulfotransfer_3 (PF13469), which have an average length of 230.1, 218.3, and 224 amino acids, respectively. According to the model information in the Pfam database, the SOT domain 1 shows an average coverage of its contributing protein sequences of $64 \%$. An average of $16 \%$ of all amino acid residues that are covered by the HMM are identical to it. The average coverage of the SOT domains 2 and 3 in their respective sequences are 67 and 47\%, with average sequence identities of 15 and $14 \%$. In addition, HMMs for more specific SOT subfamilies have been 


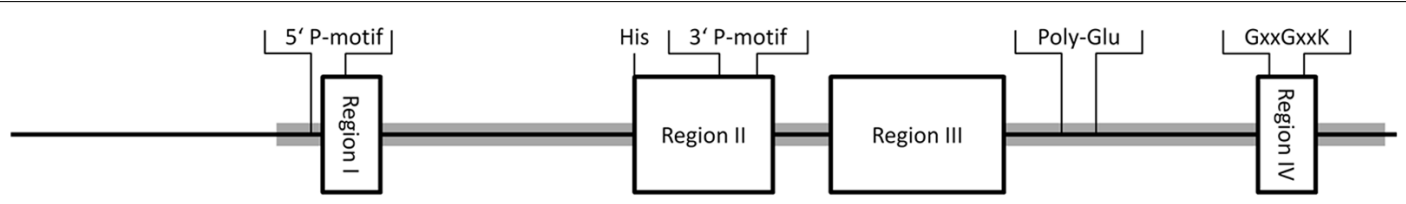

FIGURE 2 | Conserved regions I to IV of plant SOTs. Regions are shown as boxes, with size and position in the protein relative to the average size of SOTs from Arabidopsis thaliana. Functional amino acids were obtained from structural analyses of mouse SOTs as described above. The PAPS binding regions (5'P-motif, 3'P-motif, GxxGxxK), the proton acceptor histidine (His), and a poly-glutamic acid (Poly-Glu), that can be found in many plant SOTs, are labeled. The position of the Pfam domain PF00685 is shaded in gray.

Table 1 | Summary of the members of the SOT family in Arabidopsis and their putative substrates.

\begin{tabular}{|c|c|c|c|c|c|c|c|}
\hline \multirow[b]{2}{*}{$\begin{array}{l}\text { NCBI } \\
\text { accession }\end{array}$} & \multirow[b]{2}{*}{$\begin{array}{l}\text { Arabidopsis } \\
\text { gene ID }\end{array}$} & \multicolumn{3}{|c|}{ Nomenclature } & \multirow[b]{2}{*}{$\begin{array}{l}\text { Amino } \\
\text { acids }\end{array}$} & \multirow[b]{2}{*}{ Preferred substrate } & \multirow[b]{2}{*}{ Reference } \\
\hline & & I & II & III & & & \\
\hline NP_199182 & AT5G43690 & AtSOT1 & & AtSULT202B4 & 331 & & \\
\hline NP_190689 & AT3G51210 & AtSOT2 & & Pseudogene & 67 & & \\
\hline NP_190093 & AT3G45070 & AtSOT5 & AtST3a & AtSULT202B1 & 323 & Flavonol & $\begin{array}{l}\text { Gidda and Varin (2006), } \\
\text { Hashiguchi et al. (2013) }\end{array}$ \\
\hline NP_190094 & AT3G45080 & AtSOT6 & AtST3b & AtSULT202B2 & 329 & & \\
\hline NP_174139 & AT1G28170 & AtSOT7 & & AtSULT202B8 & 326 & & \\
\hline NP_565305 & AT2G03750 & AtSOT11 & & AtSULT202D1 & 351 & & \\
\hline NP_178471 & AT2G03760 & AtSOT12 & AtST1 & AtSULT202A1 & 326 & $\begin{array}{l}\text { Flavonone, brassinosteroids, } \\
\text { salicylic acid }\end{array}$ & $\begin{array}{l}\text { Lacomme and Roby (1996), } \\
\text { Marsolais et al. (2007), } \\
\text { Baek etal. (2010), } \\
\text { Hashiguchi et al. (2013) }\end{array}$ \\
\hline NP_178472 & AT2G03770 & AtSOT13 & & AtSULT202E1 & 324 & Flavonol & Hashiguchi et al. (2013) \\
\hline NP_196317 & AT5G07000 & AtSOT14 & AtST2b & AtSULT203A2 & 347 & & \\
\hline NP_568177 & AT5G07010 & AtSOT15 & AtST2a & AtSULT203A1 & 359 & Hydroxyjasmonate & Gidda et al. (2003) \\
\hline NP_177550 & AT1G74100 & AtSOT16 & AtST5a & AtSULT201B3 & 338 & $\begin{array}{l}\text { Phenylalanine and tryptophan } \\
\text { derived dsGls }\end{array}$ & $\begin{array}{l}\text { Piotrowski etal. (2004), } \\
\text { Klein etal. (2006) }\end{array}$ \\
\hline NP_190631 & AT3G50620 & AtSOT19 & & & 340 & & \\
\hline NP_179175 & AT2G15730 & AtSOT20 & & & 344 & & \\
\hline NP_195168 & AT4G34420 & AtSOT21 & & & 403 & & \\
\hline NP_563804 & AT1G08030 & TPST & & & 500 & Tyrosylprotein & Komori etal. (2009) \\
\hline
\end{tabular}

I: Nomenclature used in this review introduced by Klein and Papenbrock (2004). II: Nomenclature introduced by Piotrowski et al. (2004). III: Nomenclature introduced by Hashiguchi etal. (2013). The term TPST was introduced by Komori etal. (2009). 
deposited in Pfam. Generally, they show a lower number of hits in the database, with mostly increased sequence coverage and a higher average sequence identity as compared to the more general SOT domains 1-3. The aryl SOT domains Arylsulfotrans_1 and Arylsulfotrans_2 (PF05935 and PF14269) and the Stf0 SOT domain Sulphotransf (PF09037) represent groups of sequences with more specific occurrence, especially in prokaryota. The two aryl SOT domains show an average coverage of 83 and 57\%, with an identity of 30 and $28 \%$, respectively. For the Stf0 SOT domain the average sequence coverage is $81 \%$, with an average identity of $33 \%$. The galactose-3-O-SOT domain (PF06990) shows coverage of $78 \%$ with $24 \%$ identity.

\section{SULFOTRANSFERASE FAMILIES IN DIFFERENT PLANT GENOMES}

Sulfotransferases have a broad range of substrates and therefore many functions. In previous literature it has been stated that SOTs are present in all kingdoms except in Archaea (Klein and Papenbrock, 2008; Chen et al., 2012). Nevertheless, according to the protein family database Pfam [http://pfam.xfam.org/ (accessed 23.06.2014)] there are Archaea sequences with a characteristic conserved SOT domain.

There are only few studies that aim to identify all SOTs of a plant species. A requirement to do this is a fully sequenced genome, but due to their eclectic functions, we assume that SOTs are present in almost every plant species. The Pfam database already stores 538 putative plant SOT sequences, 459 of which have a Sulfotransfer_1 domain (PF00685), 49 a Sulfotransfer_2 domain (PF03567), 16 a Sulfotransfer_3 domain (PF13469), and 10 a Sulphotransf domain (PF09037). The actual number might be less, because of redundant entries. The Sulfotransfer_3 (PF13469) and the Gal-3-O_sulfotr domain (PF06990) are only present in algae. The Arylsulfotrans domain (PF05935) is not present in plants, while Arylsulfotran_2 (PF14269) is only found in a single Ricinus communis L. sequence.

While so far 22 putative A. thaliana SOTs were identified, 35 genes coding proteins with a SOT domain were reported in Oryza sativa L., including six genes likely to be pseudogenes (Chen et al., 2012). In phylogenetic analyses, they are clustered into seven subfamilies. However, microarray data revealed that the genes within subfamilies are expressed in a different manner, indicating individual functions. When 17 AtSOTs were added to the distance trees, they did not group together with any of the O. sativa genes. This was taken as a hint for independent evolution of $O$. sativa and $A$. thaliana SOTs by gene duplication or loss. This was supported by the finding that half of the O. sativa SOTs contain introns, which is hardly the case for AtSOTs (Klein and Papenbrock, 2004).

Comparative genomics studies were conducted in Brassica rapa L. with A. thaliana, in order to identify all Gl biosynthesis genes (Zang et al., 2009; Wang et al., 2011). Thirteen putative desulfoglucosinolate SOT ( $d s G l$ SOT) genes were identified. Two genes are paralogs of AtSOT16, 1 of AtSOT17, and 10 of AtSOT18. One AtSOT18 paralog appears to be nonfunctional, because of transposon insertion, and one carries a frame shift. None of the genes contains introns, as it is the case for AtSOT genes. All paralogs share at least $70 \%$ sequence identity with their AtSOT counterparts, with the exception of one SOT from B. rapa (BrSOT18, 68\%; Wang etal., 2011). The higher number of BrSOTs is explained by the triplication of the $B$. rapa genome and later duplication, transposition, or tandem duplication of the genes.

In Brassica napus L., so far only twelve putative genes encoding SOTs were identified (Rouleau et al., 1999; Marsolais et al., 2000). Additionally, there are at least five isoforms of dsGl SOTs in $B$. napus, which have similar substrate affinities as their A. thaliana homologs (own unpublished results). Regarding that B. napus is an allotetraploid species formed by the hybridization of $B$. rapa and $B$. oleracea, a much higher number of SOT genes can be expected.

To group these diverse enzymes into families and subfamilies remains a difficult task. Klein and Papenbrock (2004, 2008) ordered $21 \mathrm{~A}$. thaliana SOTs in eight groups, according to their amino acid sequence identity. However, already characterized SOTs with the same substrate specificity did not group together, and even high sequence identity of more than $85 \%$ among two SOTs did not reveal equal enzymological characteristics. Neither sequence identity, nor generated trees ordered already characterized SOTs in groups according to their substrate specificities. Three dsGI SOTs were on one separate branch, but flavonoid and brassinosteroid SOTs could not be distinguished.

Hernàndez-Sebastiá et al. (2008) generated a phylogenetic tree including 78 SOTs from 13 different plant species. This approach faced the same problems as the one by Klein and Papenbrock $(2004,2008)$ and it was again concluded that the prediction of SOT substrates by high primary sequence identities is limited. For example, it was speculated that AtSOT13 was a brassinosteroid SOT, because of its close distance to AtSOT12, but Hashiguchi et al. (2013) showed that AtSOT13 uses flavonoids as preferred substrates.

Another attempt included, besides 17 A. thaliana, also B. napus and Flaveria spp. sequences (Hashiguchi et al., 2013). AtSOT2 was excluded, because it is most likely a pseudogene. According to a dendrogram, three families were defined with two, three and five subfamilies, respectively. The families had an amino acid sequence identity of at least $45 \%$ and the subfamilies of at least $60 \%$. But again, except for the dsGl SOTs, the SOTs did not group together according to their substrate specificities.

Labonne et al. (2009) tried to identify a putative SOT of Turnera krapovickasii Arbo (Passifloraceae) by phylogenetic analysis. The sequence was aligned with 28 SOTs from A. thaliana, B. napus, Vitis vinifera L., O. sativa, Hordeum vulgaris L., Populus trichocarpa Hook. The SOT from T. krapovickasii was on a branch by itself and alignments with characterized SOTs revealed low sequence identity. Therefore, it was not possible to identify the function of the respective SOT.

Overall, past attempts indicate that it is difficult to order plant SOTs according to their amino acid sequence. Only for dsGl SOTs does it seem to be possible, because they are clustered together on a separate branch in all approaches. Therefore, only enzymatic assays with additional mutational studies can give reliable information about substrate specificity and function.

\section{SUBSTRATES FOR SULFOTRANSFERASES BIOSYNTHESIS OF THE CO-SUBSTRATE PAPS}

$3^{\prime}$-phosphoadenosine $5^{\prime}$-phosphosulfate is an obligate cosubstrate for sulfation reactions catalyzed by SOTs. In plants, PAPS does not represent an intermediate of reductive sulfate assimilation 
as in fungi and some bacteria, but it seems to play an exclusive role as a sulfuryl donor for sulfation reactions. PAPS is synthesized from ATP and sulfate in a two-step reaction (Figure 3). In the first step, ATP sulfurylase (EC 2.7.7.4) catalyzes sulfate activation. The enzyme hydrolyses the bond between the $\beta$ - and the $\gamma$-phosphates of ATP and then adds sulfate to the $\gamma$-phosphate. The activation step is necessary, because sulfate is metabolically inert. The energy is stored in the phosphoric acid-sulfuric anhydride bond of the reaction product, adenosine $5^{\prime}$-phosphosulfate (APS), allowing sulfate to undergo further reactions. The energetic balance of the sulfate adenylylation reaction favors ATP formation. Therefore, the reaction products, APS, and pyrophosphate $\left(\mathrm{PP}_{\mathrm{i}}\right)$, need to be maintained at a low concentration by the enzymes inorganic pyrophosphatase that hydrolyses $\mathrm{PP}_{\mathrm{i}}$, APS reductase (EC 1.8.4.9) and APS kinase (EC 2.7.1.25; AKN) that metabolize APS. APS reductase catalyzes the first step of sulfate reduction. APS kinase catalyzes the ATP-dependent phosphorylation on the $3^{\prime}$-position of APS. In vitro tests have shown that excess APS inhibits APS kinase. The product PAPS is the substrate for the SOT proteins.

In general, the availability of PAPS for sulfation in vivo depends on its synthesis, transport, degradation, and utilization as investigated in mammals (Klaassen and Boles, 1997). Recently, it was shown that the transporter PAPST1 in the chloroplast envelope membrane is not only involved in the provision of PAPS for the extraplastidic sulfation reactions, but is also capable to transport PAP in an antiport manner. The loss of PAPST1 leads to a decreased production of sulfated compounds like Gl, increased production of $\mathrm{dsGl}$, and the modulation of primary sulfate assimilation, another indication for the strong interconnectedness of primary and secondary sulfur metabolism (Gigolashvili et al., 2012). The by-product of the sulfation reaction, PAP, has gene regulatory attributes. In turn, PAP is regulated by the adenosine bisphosphate phosphatase SAL1 that dephosphorylates PAP to adenosine monophosphate. 3'-Phosphoadenosine 5'-phosphate accumulates at drought stress and high light conditions. Mutational studies indicated that PAP inhibits $5^{\prime}-3^{\prime}$ exoribonucleases in the cytosol and nucleus, which causes changes in expression of stressresponsive genes. It was suggested that a PAP-SAL1 retrograde pathway alters gene expression as part of the stress response

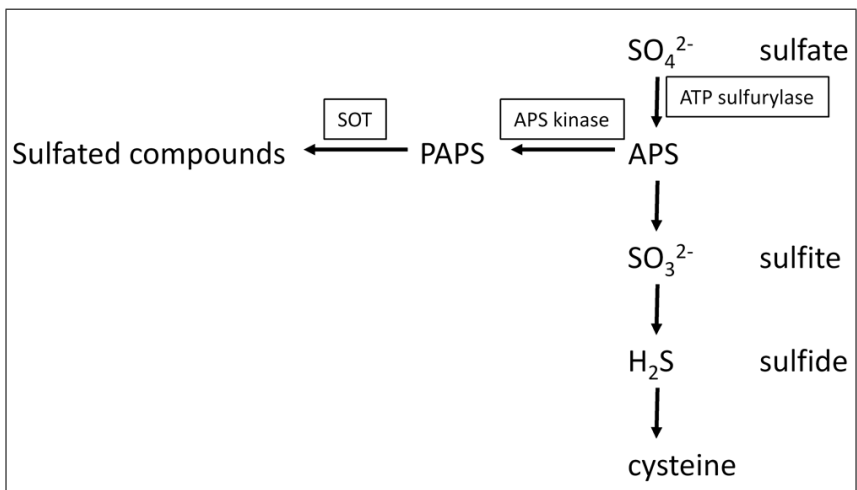

FIGURE 3 | Biosynthesis of APS and PAPS and the connection of primary and secondary sulfur metabolism.
(Estavillo et al., 2011). Additionally, a correlation between the increases of PAP with changes in the sulfur metabolism was reported. Further analysis of sall knock out mutants led to the conclusion that changes of gene expression due to sulfur limitation is triggered by internal sulfur deficiency and not by low external sulfur levels. PAP accumulation also resulted in an increase of enzymatic oxygenation of fatty acids, an increase of jasmonic acid synthesis and a decrease of Gls. Possible explanations for the Gl decrease could be inhibition of dsGl SOTs or the disruption of PAPS transport from plastids to the cytosol (Gigolashvili et al., 2012; Lee et al., 2012).

\section{SUBSTRATES FOR PLANT SOTs}

The first isolated and characterized plant SOTs were flavonol $3^{\prime}$ - and flavonol 4'-SOT of Flaveria chloraefolia (Varin et al., 1992) and later of F. bidentis (L.) Kuntze (Varin et al., 1997b). These SOTs sequentially sulfate specific hydroxyl groups of the flavonol quercetin to quercetin tetrasulfate. Flavonol biosynthesis was demonstrated to be regulated by auxin and ethylene. In turn, the flavonol quercetin and quercetin sulfates affect root development processes, such as the basipetal root auxin transport, elongation growth, and gravitropism (Faulkner and Rubery, 1992; Lewis et al., 2011).

So far, four flavonoid SOTs have been characterized in $A$. thaliana: AtSOT5, AtSOT8, AtSOT12, and AtSOT13 (see Table 1 for details). Hashiguchi et al. (2013) compared characteristics and substrate specificities of AtSOT5, AtSOT12, and AtSOT13.

AtSOT13 and AtSOT5 showed the highest activity with the flavonol galangin (3,5,7-trihydroxy-2-phenyl-4H-chromen4-one), while AtSOT12 showed the highest activity for the flavanone naringenin [(2S)-5,7-dihydroxy-2-(4-hydroxyphenyl)2,3-dihydro-4H-chromen-4-one] and was the only SOT that sulfates anthocyanidin. Interestingly, the AtSOTs showed no or comparably low activity with quercetin. It was speculated that the position-3 hydroxyl group of quercetin inhibits the catalytic activity. It was also shown that only AtSOT12 is able to use 3hydroxyflavone as substrate, while 7-hydroxyflavone is used by all three AtSOTs. After comparisons of $K_{\mathrm{m}}$-values using kaempferol as substrate, it was concluded that particular hydroxyl groups of kaempferol are specifically sulfated by the AtSOTs.

AtSOT8 was also characterized by Hashiguchi et al. (2014). The $\mathrm{pH}$ optimum at 5.5 was lower than for previously characterized flavonoid SOTs. Thus it was speculated that AtSOT8 might be located in the vacuole. Comparison of $V_{\max } / K_{\mathrm{m}}$-values showed that AtSOT8 prefers flavonol glycosides instead of their aglycone counterparts as substrates. Also, there was only activity to flavonoids with a hydroxyl group at position 7. Hence, it was suggested that AtSOT8 might be a flavonol glucoside-7 SOT. Surprisingly, neither sulfated glucoside flavonoids could be detected in vivo in A. thaliana by LC/MS, nor were there any accordant database entries. Possible explanations for the non-detected sulfated glucoside flavonoids could be low or condition-dependent occurrence.

AtSOT10 showed activity with brassinosteroids (Marsolais et al., 2007), specifically brassinosteroid biosynthetic end products. In summary, it was speculated that it inactivates brassinosteroids and therefore is involved in plant development processes. In 
numerous studies, overexpression of brassinosteroid catabolic genes led to brassinosteroid-deficient phenotypes. However, overexpression and T-DNA insertion in null mutants of AtSOT10 did not show brassinosteroid-deficient phenotypes, emphasizing difficulties of transferring in vitro results to in vivo insights (Sandhu and Neff, 2013).

Of all investigated A. thaliana SOTs so far, AtSOT12 has the broadest substrate specificity. Besides using flavonoids as substrates, it was also shown to be active with brassinosteroids and salicylic acid. Within the brassinosteroids, it showed preference for 24-epibrassinosteroids (Marsolais et al., 2007). It was stereospecific for 24-epibrassinosteroids and accepted mammalian hydroxysteroids and estrogens, too. The most preferred substrate was the metabolic precursor 24-epicathasterone $\left(K_{\mathrm{m}}=6.9 \mu \mathrm{M}\right)$, which showed inhibitory effects above $5 \mu \mathrm{M}$. The $K_{\mathrm{m}}$-value for salicylic acid is comparably high (440 $\mathrm{MM}$; Baek et al., 2010). Salicylic acid is a signal molecule in plant defense, and cellular concentrations increased up to $40 \mu \mathrm{M}$ after pathogen infection indicating that sulfation of salicylic acid is a response to pathogen attack. This theory was supported by the fact that atsot 12 knock out mutants were less resistant to the pathogen Pseudomonas syringae, while AtSOT12 overexpressing lines showed a higher resistance.

Two B. napus brassinosteroid isoforms, BNST3 and BNST4, were enzymatically characterized. Recombinant BNST3 stereospecifically sulfated 24-epibrassinosteroids and preferred 24epicathasterone $\left(\mathrm{K}_{\mathrm{m}}=1.4 \mu \mathrm{M}\right)$, which is a biosynthetic intermediate of 24-epibrassinolide. Because of the biological inactivity of 24-epibrassinolide sulfate, it was hypothesized that BNST3 is involved in brassinosteroid inactivation (Rouleau etal., 1999). BNST4 also preferred 24-epibrassinosteroids $\left(K_{\mathrm{m}}=4.9 \mu \mathrm{M}\right)$, but also showed a broad substrate specificity with other steroids, also indicating a role in detoxification (Marsolais et al., 2004). Overall, they showed similar substrate specificities toward brassinosteroids as AtSOT12 (Marsolais et al., 2007).

AtSOT15 specifically sulfates 11 - and 12-hydroxyjasmonate, which is a signaling molecule in plant defense and development. $K_{\mathrm{m}}$-values indicate a higher affinity to 12 - than to 11-hydroxyjasmonate (10 $\mu \mathrm{M}$ and $50 \mu \mathrm{M}$, respectively). 12hydroxyjasmonate naturally occurs in A. thaliana and it was suggested that sulfation might function in inactivation of 12hydroxyjasmonic acid (Gidda et al., 2003).

Komori et al. (2009) identified a 62 kDa, Golgi-localized, transmembrane protein, that sulfates tyrosylproteins in A. thaliana. The recombinantly expressed TPST sulfated tyrosine residues of precursor polypeptides of the "plant peptide containing sulfated tyrosine 1" (PSY1) and phytosulfokine (PSK). PSY1 and PSK are peptide hormones, which promote growth and cell proliferation (Matsubayashi and Sakagami, 1996; Amano et al., 2007). The activity with both substrates indicates broad substrate specificity. TPST showed a higher activity with PSY1, which was explained by a closer proximity of an acidic region to the sulfated tyrosine residue. TPST loss-of-function mutants showed numerous abnormal attributes, which led to the conclusion that sulfated peptides or proteins are involved in plant growth and development. Previously, in microsomal membrane preparations from carrot cells, rice, and asparagus TPST activity was shown
(Hanai etal., 2000). In rice, the $K_{\mathrm{m}}$-value was $71 \mu \mathrm{M}$ at a $\mathrm{pH}$ of $7.0-8.5$ in the presence of manganese ions. The enzyme kinetic values such as $K_{\mathrm{m}}$ and $V_{\max } A$. thaliana TPST remain to be determined. TPST also sulfates peptide root meristem growth factors (RGFs), which are involved in postembryonic root development. Loss-of-function tpst-1 mutants showed reduction in root meristem size and loss of coordination between cell elongation and expansion in the elongation-differentiation zone. Addition of RGF restored the meristem activity to $\sim 70 \%$ and addition of RGF, PSK, and PSY1 restored the activity comparable to the wild-type. Sulfation of RGFs was found to be critical for its function. Further experiments showed that RGFs positively regulate the expression of PLETHORA transcription factors that mediate the pattern of the root stem niche (Matsuzaki et al., 2010).

Other examples of already characterized SOTs like a choline-Osulfate SOT of the halophytic Limonium species (Rivoal and Hanson, 1994) and a plasma membrane-associated gallic acid SOT of M. pudica L. (Varin et al., 1997a) undercut the diversity of substrates and functions of these enzymes. Choline sulfate is an osmolyte that accumulates under saline conditions. The respective choline-O-SOT showed a fourfold higher activity under high salinity. The choline-O-SOT had its $\mathrm{pH}$ optimum at 9.0 and the $K_{\mathrm{m}}$-value for choline was $25 \mu \mathrm{M}$. The $42 \mathrm{kDa}$ membrane bound gallic acid SOT might be involved in the regulation of the seismonastic response. It showed strict substrate specificity and a $\mathrm{K}_{\mathrm{m}}$-value of only $3.0 \mu \mathrm{M}$.

Further studies indicate the existence of more SOTs, even though they were not especially isolated or characterized. It was shown in vivo that poplar trees convert hydroxylated metabolites of polychlorinated biphenyls (PCBs) into sulfated PCB. It was suggested that SOTs catalyze this reaction (Zhai et al., 2013). Sulfated polysaccharides occur in marine angiosperms, mangroves (Aquino et al., 2005, 2011), freshwater plants (Dantas-Santos et al., 2012), and algae (Ngo and Kim, 2013), which are likely to be sulfated by not yet identified SOTs.

\section{GLUCOSINOLATES: PRODUCTS OF THE SOT REACTION}

Glucosinolates are a group of over 200 nitrogen- and sulfurcontaining natural products found in vegetative and reproductive tissues of 16 plant families within the Capparales (Clarke, 2010). They are well-known as the major secondary metabolites in agriculturally important crop plants of the Brassicaceae family, such as oilseed rape (B. napus), fodder and vegetables (e.g,. broccoli and cabbage). The model plant $A$. thaliana. Gl share a core structure containing a $\beta$-D-glucopyranose residue linked via a sulfur atom to a $(Z)-N$-hydroximino sulfate ester. They are distinguished by a variable $\mathrm{R}$ group derived from one of several amino acids, mainly tryptophan, phenylalanine and methionine (Mithen, 2001). The Gl pattern varies among the plant species and among $A$. thaliana ecotypes. In 39 A. thaliana ecotypes, 34 different Gls have been identified. Quantity and composition of Gls depend on the developmental stage of the plants and on the plant organ (Kliebenstein et al., 2001a).

Intact Gls are not toxic to cells. However, after cell damage Gls are hydrolyzed, catalyzed by thioglucosidase enzymes ("myrosinase"), to produce a variety of volatile hydrolysis 
products, such as thiocyanates, isothiocyanates, and nitriles. Only these breakdown products have a wide range of biological activities including both negative and positive effects (Fenwick and Heaney, 1983). In several studies these breakdown products were shown to be involved in plant defense against pathogens and herbivores. Thus, Gls are the best-characterized preformed defense compounds in the Brassicaceae and contribute to the protection against pathogens of the generalist type (Rausch and Wachter, 2005).

The last step in the Gl core structure biosynthesis of the different aliphatic, aromatic, and indole desulfo (ds) Gls is catalyzed by members of the SOT family. Glendening and Poulton (1990) partially purified a protein from Lepidium sativum L. that had PAPS-dependent dsGl SOT activity; however, at that time no molecular data was available. Later it was shown that three SOT proteins from $A$. thaliana are involved in Gl biosynthesis catalyzing the sulfation of dsGls to the intact Gls (Varin and Spertini, 2003; Piotrowski et al., 2004; Hirai et al., 2005).

\section{Sulfotransferases involved in sulfation of desulfo-glucosinolates}

The three dsGl AtSOT proteins (AtSOT16, AtSOT17, and AtSOT18) were predicted and then verified by different means (screening of many sulfated compounds, combining of knowledge, and integration of metabolomics and transcriptomics) for being responsible for the sulfation of dsGl (Varin and Spertini, 2003; Piotrowski et al., 2004; Hirai et al., 2005). Up till now, it was not unambiguously demonstrated why multiple $d s G l$ SOT genes have been conserved during evolution in A. thaliana and in other Brassicaceae species.

The glucosylation and the sulfation reactions were assumed to be non-specific with respect to the side chain (Halkier, 1999). It is also hypothesized that first the side chains are elongated to synthesize so-called parent Gls, then the glycone moiety is developed and finally, several side chain modifications take place to produce the respective daughter Gl (Wittstock and Halkier, 2002). However, it is not clarified when the dsGls are sulfated by SOT proteins and whether there is a specificity for certain parent or daughter Gls. As the Gl pattern differs among $A$. thaliana ecotypes (Kliebenstein et al., 2001a), the investigation of the three dsGl SOTs from ecotype C24, which shows the broadest variety of Gls in comparison to other ecotypes, was most rational. In addition, one exemplary SOT from the fully sequenced ecotype Col- 0 was investigated. To determine if and how these three dsGl SOT proteins might influence the Gl pattern, different in vitro enzyme assays were performed. Substrate specificity varies among the three proteins in the same ecotype (C24) and between ecotypes (C24 versus Col-0). AtSOT16 (C24) has the broadest substrate specificities. Tryptophan and phenylalanine-derived dsGl are the most preferred substrates, but it also accepts methionine-derived dsGl of chain length $\mathrm{C} 3$, C4, C5, C6, C7, and C8, although at much lower activities. AtSOT17 (C24) has narrow substrate specificities and does not act upon tryptophan-derived dsGl. Phenylalanine-derived benzyl dsGl is the most preferred substrate, but it also accepts methionine-derived dsGls, but has a strong preference for longer side chains, C6, C7, and C8. AtSOT18 (C24) also has narrow substrate specificities, does not act upon tryptophan-derived dsGl.
It accepts phenylalanine-derived dsGl and methionine-derived $\mathrm{dsGl}$, but has a strong preference for longer side chains, C6, $\mathrm{C7}$, and C8. In summary, these three enzymes differ significantly in their affinity for the investigated substrates and the co-substrate PAPS (Klein etal., 2006; Klein and Papenbrock, 2009). It was speculated that the differences between AtSOT1618 could be an explanation for the different Gl patterns between organs, developmentally stages and growth environments reported by Brown etal. (2003). Anyhow, Møldrup et al. (2011) transformed genes involved in Gl biosynthesis into tobacco, thus successfully enabling it to synthesize Gls. In this approach, they could show that SOTs are not the bottleneck of Gl synthesis, but the supply of the co-substrate PAPS. Therefore, regulation of AtSOT16-18 could be, only taken together with other Gl and PAPS biosynthesis genes, partly responsible for Gl variation.

Up to now, the knowledge on secondary modifications of parent Gls is limited (Graser et al., 2001). In future work it could be interesting to verify the general acceptance that parent dsGls are sulfated before secondary modifications of Gls take place (Kliebenstein et al., 2001b). However, assuming the general acceptance is right, no secondarily modified Gls would exist in a ds form to interact with the SOTs. Therefore, it is possible, that artificially de-sulfated Gls with secondary modifications are sulfated in vitro, but with no in vivo relevance.

\section{In A. thaliana ecotypes SOT18 proteins differ in their sequence and substrate specificity}

It was shown that AtSOT18 proteins from two different $A$. thaliana ecotypes differ in their kinetic parameters as well as their substrate specificities. The primary structure of AtSTO18 proteins from the ecotypes Col-0 and C24 differ in two amino acids (Klein et al., 2006; Klein and Papenbrock, 2009). One could assume that there could be a correlation of AtSOT18 enzyme activities and differences in Gl profiles between these ecotypes. Therefore, AtSOT18 sequences from eight $A$. thaliana ecotypes with highly diverse Gl patterns were investigated: The AtSOT18 sequence from Col-0 showed the highest similarity to the largest number of other sequences in the alignment. The AtSOT18 proteins showed sequence deviations of maximal two amino acids in comparison to the AtSOT18 sequence from Col-0. The positions of the amino acid replacements were different in each sequence. The small differences in the primary sequence lead to important structural changes in secondary and tertiary structure that might be the key for different kinetic activities toward a broad range of substrates (Luczak et al., 2013). All recombinant AtSOT18 proteins showed low substrate specificity with an indolic Gl, while the specificity for aliphatic substrates varied. There was no correlation in the kinetic behavior with the major dsGl contents or with the ratio of $\mathrm{C}_{3} / \mathrm{C}_{4} \mathrm{dsGl}$ in the respective ecotype. Therefore, it is unlikely that dsGl AtSOT18 enzymes play a major role in shaping the Gl profile in A. thaliana (Luczak et al., 2013). Interestingly, in humans, inter-individual variation in sulfation capacity may be important in determining an individual's response to xenobiotics, and recent studies have begun to suggest roles for SOT polymorphism in disease susceptibility (Gamage et al., 2006). Variations in concentration and composition of Gls in A. thaliana ecotypes 
and different environmental conditions can still neither be fully explained, nor predicted.

\section{EXPRESSION OF SULFOTRANSFERASES}

Sulfated compounds are mainly linked to biotic and abiotic stress response. This is supported by several expression studies of SOTs. So far, the mRNA levels of most characterized A. thaliana SOTs were rather low under normal growth conditions (Lacomme and Roby, 1996; Gidda et al., 2003; Piotrowski et al., 2004). This is supported by the fact that there is a relatively low number of SOTs in EST databases with the exception of AtSOT15 and AtSOT16 (Klein and Papenbrock, 2008). However, the expression of AtSOT12, AtSOT15, AtSOT16, and AtSOT17 was significantly increased by treatment with jasmonate (Lacomme and Roby, 1996; Gidda et al., 2003; Piotrowski et al., 2004).

Only 17 AtSOTs were found to be present on $24 \mathrm{k}$ Affymetrix chips and for many of those the absolute signal was quite low (Klein and Papenbrock, 2008). Hashiguchi et al. (2013, 2014) reported that microarray database research suggested that AtSOT8 is mainly expressed in roots, while AtSOT13 is expressed in the early stages of the embryonic development. Interestingly, Hashiguchi et al. (2013) cloned AtSOT13 from 2-week old seedlings.

Transcripts of AtSOT10 were mainly detected in roots. Transcript levels were repressed $4 \mathrm{~h}$ after trans-zeatin treatment. After $8 \mathrm{~h}$ no transcripts were detectable anymore by qRT-PCR (Marsolais et al., 2007).

Northern Blot analysis revealed that AtSOT12, the encoded protein uses brassinosteroids, flavonoids, and salicylic acid as substrates, is moderately expressed in roots and leaves, and highly in flowers, while no expression was detected in stems and siliques. Furthermore, it was strongly induced by salt and sorbitol and slightly by cold, ABA, auxins, cytokinins, methyl jasmonate, salicylic acid, and interactions with bacterial pathogens (Lacomme and Roby, 1996; Baek et al., 2010). These results strongly indicate a function of AtSOT12 in stress and hormone response. Similar results were obtained for the respective homologous genes in B. napus. BNST3 and BNST4 mRNA levels were quite low, but increased after treatment with salicylic acid, ethanol, xenobiotics, low oxygen stres, and the herbicide safener naphtalic anhydride (Rouleau et al., 1999; Marsolais et al., 2004). BNST3 and BNST4 induction also indicates a function in stress response and detoxification.

The protein encoded by AtSOT15 uses hydroxyl jasmonate as substrate. Expression was induced upon methyljasmonate and 12-hydroxyjasmonate treatment. Probably, it inactivates the function of jasmonic acids and therefore enhances the hypocotyl growth (Gidda et al., 2003). Yamashino et al. (2013) showed that AtSOT15 transcription is also regulated by an external coincidence mechanism. Database research indicated that AtSOT15 might be a target of the phytochrome interacting transcriptional factors PIF4 and PIF5. PIF4 and PIF5 are controlled by the circadian clock, but also independently influenced by light and temperature. Further qRT-PCR analysis showed that AtSOT15 was diurnally regulated by PIF4 and PIF5 at the end of a short day dark phase and/or high temperatures. Accordingly, AtSOT15 is induced under conditions when hypocotyl growth takes places.
At first, the dsGl SOTs AtSOT16-18 were reported to be constitutively expressed in all leaves, flowers, and siliques (Varin and Spertini, 2003). Later Northern Blot analysis revealed that AtSOT16 mRNA level increased after treatment with coronatine (an analog of octadecanoid signaling molecules), jasmonic acid precursor 12-oxophytodienonic, ethylene precursor ACC and after treatment with jasmonic acid. UV-C illumination and wounding also induced AtSOT16 expression. AtSOT17 mRNA increased 2.4 fold and 1.2 fold, respectively, while AtSOT17 expression only slightly increased ( 1.3 fold) after coronatine treatment (Piotrowski et al., 2004). Regarding the developmental stages, AtSOT16 and AtSOT17 mRNA levels were highest in two week old seedlings and lowest in flowering plants. In contrary, AtSOT18 levels were quite low in young plants and slightly increased after 5-6 weeks. Only AtSOT17 expression was influenced by a 12 hour dark / 12 hour light cycle. It was the highest at the end of the light phase and the lowest at the end of the dark phase. No differences in any of the three mRNA levels were detected, when A. thaliana was grown in media with tenfold sulfate concentration (Klein et al., 2006).

Huseby et al. (2013) investigated how the Gl biosynthesis is controlled by light and the diurnal rhythm. By qRT-PCR analyses, it was shown that AtSOT16, AtSOT17, and AtSOT18 are up regulated in light and down regulated in darkness. Further experiments indicated that the three dsGl AtSOTs are controlled by different transcriptional factors. In $A$. thaliana mutants, lacking the transcription regulator HY5, AtSOT18 was less up-regulated than in the wild-type, indicating the HY5 is in control of AtSOT18. Interestingly, HY5 not only promotes numerous genes, but also seemed to repress MYBs. MYBs are a group of transcription factors, which are also involved in the control of Gl biosynthetic genes (Gigolashvili et al., 2007a,b, 2008; Hirai et al., 2007; Sønderby et al., 2007; Malitsky et al., 2008; Sønderby et al., 2010; Li et al., 2013; Jensen et al., 2014). AtSOT16 was significantly down regulated in myb34 myb51 myb122-2 triple mutant, revealing the specific control of these transcription factors (Frerigmann and Gigolashvili, 2014). Furthermore, MYB51, an indolic Gl metabolism specific transcription factor, was found to be down regulated in the dark, resulting in repression of indolic dsGl specific AtSOT16. Another indolic Gl transcription factor, MYB34, was up regulated after re-illumination (Celenza et al., 2005). This was not the case for MYBs controlling aliphatic Gl biosynthesis. It was concluded that $M Y B$ factors controlling biosynthesis of indolic Gl have a specific function in light regulation of their target gene, unlike the aliphatic group of $M Y B$ (Huseby et al., 2013).

Even so, the interaction and hierarchy of HY5 and MYBs still remains unclear. AtSOT16, AtSOT17, and AtSOT18 were also up regulated in apk1 apk2 double mutants. Hence, a reduction in PAPS supply and therefore reduction in Gl concentration leads to an up regulation $d s G l$ SOTs (Mugford et al., 2009).

The expression of the twelve putative $d s G l$ SOTs in B. rapa was investigated by qRT-PCR (Zang et al., 2009). Two genes are paralogs of AtSOT16, one of AtSOT17, and ten of AtSOT18. Generally, BrSOT16s were most strongly expressed, followed by BrSOT18s and then the BrSOT17s. With the exception of one BrSOT18, all of them were expressed in all examined tissue 
types. One BrSOT16 was expressed in all tissue types, except in the stamen, while the other one was strongly expressed in the stamen, but weakly in the floral bud and carpel. Some BrSOT18s were strongly expressed in the carpel and others in the stamen. Hence, the expression was not tissue-specific, but there was great variation in between tissue types. The expression of some BrSOT18s was developmentally regulated, but not of BrSOT16s. Again it was concluded that the expression could influence the Gl content, since SOTs play a crucial role in Gl biosynthesis.

The TPST gene is expressed in the whole plant, which was shown by analyzing A. thaliana TPST-GUS transformants, but especially strong in the root apical meristem and in the lateral root primordial and vascular tissues (Komori et al., 2009).

Expression of the 35 O. sativa SOTs was investigated by microarray database analysis (Chen et al., 2012). The overall expression was reported to be considerably low. Low expression levels were in the apical meristem and young leaves. Higher expression was found in the stigma, ovary and roots. Treatment with IAA and BAP led to up and down regulation of several SOTs also with differences in respect to tissue types and seedlings age. Furthermore, expression of eleven SOTs reacted to abiotic stress, such as high and low temperatures and dehydration. It was concluded that the individual responses of SOTs indicate functions in stress response and plant development.

Overall, SOT expressions suggest functions in plant defense, stress response, signaling and developmental regulation. Sulfation can either lead to activation or deactivation of the according substrate. SOT expression takes place basically in all organs and many stages in plant development. Interestingly, all SOTs studied so far, were induced by several conditions or stress signaling compounds, indicating a general stress response. Additionally, in the case of $d s G l$ AtSOTs and AtSOT15, a diurnal and circadian control was detected. It seems plausible that this could be the case for other SOTs, too.

\section{WHAT IS KNOWN ABOUT THE REACTION MECHANISM OF SULFOTRANSFERASES}

So far, the reaction mechanism of plant SOTs remains largely unknown. Kinetic and inhibition studies of a flavonol $3^{\prime}$-SOT from F. chloraefolia A. Gray led to the hypothesis of an ordered Bi-Bi mechanism (Varin and Ibrahim, 1992). However, the few conducted experiments are not sufficient enough for a definite conclusion.

More information is available about human SOTs. By presteady state binding studies, isotopic trapping, quenched-flow, and classic inhibition studies, Wang et al. (2014) completely solved the kinetic mechanism of the human SOT SULT2A1. SULT2A1 sulfates dehydroepiandrosterone and regulates binding of steroids to their receptors and detoxifies steroid-like xenobiotics. The according mechanism was found to be rapid equilibrium random. In this mechanism, substrates are bound and products are released in a random order. The ligands are bound in separate binding sites and released independently of the presence of its partner, hence without contribution of sulfuryl-group interactions. Ligand-binding rate constants also indicated that ligand-protein interactions, which enable the chemical reaction, are either established prior to addition of the second substrate and/or they are engaged as the system moves toward the transition state. Furthermore, it was shown that the release of the PAP nucleotide is the rate-determining step of the reaction. Substrate inhibition was explained by trapping of PAP in a dead end complex (enzyme with bound PAP and substrate), which decreases the release of PAP. Since closely related enzymes often share the same mechanism, it was speculated that this could also be the case for other human SOTs. Anyhow, this cannot be done for plant SOTs without further experimental analysis. This is already illustrated, when regarding that SULT2A1 is a half-site reactive dimer, while yet investigated plant SOTs are monomers.

The mechanism of a monomeric SOT Stf0 from Mycobacterium tuberculosis was analyzed by electrospray ionization mass spectrometry and Fourier transform ion cyclotron resonance mass spectrometry (Pi et al., 2005). Stf0 forms trehalose sulfate, which is the core disaccharide of the potential virulence factor sulfolipid-1. Interestingly, the results also indicated a rapid equilibrium random mechanism, at which the sulfuryl group is transferred in the ternary complex. Again, there is one binding site for products and one independent binding site for substrates. Results also indicated that PAPS binding was competitively inhibited by PAP.

Further studies of non-herbal SOTs, human estrogen SOT (Zhang et al., 1998) and insect retinol dehydratase (Vakiani et al., 1998), also indicated random Bi-Bi mechanisms. So far, only for a Rhizobium meliloti NodH SOT a hybrid random ping-pong mechanism was suggested (Pi et al., 2004). Therefore, the investigation of the complete kinetic mechanisms of plant SOTs remains an interesting task, which could also give new insights of the overall evolution of SOTs.

\section{HOW TO IDENTIFY THE SUBSTRATE SPECIFICITY? CHANCES AND RESTRICTION OF MODELING}

Simple online tools like SWISS-MODEL do not lead to satisfying Z-scores and therefore unreliable models. However, Cook et al. (2013) generated significant models of human SOTs by using more advanced programs such as MODELLER, GOLD, GROMACS, and AMBER. Models of human SOTs SULT1A1 and SULT1A2, which are Phase II detoxifying enzymes, were used for in silico docking studies. As substrates, 1455 small molecule drugs were tested. For SULT1A1, 76 substrates were predicted, of which 53 were already known substrates. Of the remaining 23 putative substrates, 21 were tested in enzyme assays and all of them were accepted as substrates. Of 22 predicted substrates for SULT2A1, eight were not previously mentioned in literature. Enzyme assays were carried out with four of the eight newly identified substrates, and all of them were accepted as substrates. For both SOTs neither a single false positive nor a false negative prediction occurred. Furthermore, 136 SULT1A1 and 35 SULT2A1 inhibitors were predicted. Two of those were exemplary tested in classical inhibition studies and both showed inhibitory effects.

But can these techniques be transferred to plant SOTs? Principally they could be transferred to plant SOTs, but it has to be kept in mind that SULT1A1 and SULT1A2 are extensively studied SOTs. Building reliable models for in silico docking studies requires 
knowledge about structure and mechanism of the protein. For example, SULT1A1 and SULT1A2 have a site cap, which regulates substrate specificity (Cook et al., 2012). This also had to be considered when generating the in silico models. Furthermore, it was shown that human SOTs have a high plasticity (Allali-Hassani et al., 2007; Berger etal., 2011) and that PAP binding leads to dramatic conformational changes, such as pre-formation of the acceptor binding pocket (Bidwell et al., 1999; Dajani et al., 1999; Berger et al., 2011). Hence, without prior structural knowledge about at least some of the plant SOTs, in silico modeling is still restricted. Nevertheless, in silico modeling of SOTs is a promising approach, especially because of the limitations in substrate identification based on phylogenetic analyses.

\section{A. THALIANA AS A MODEL PLANT - SUITED FOR THE ELUCIDATION OF ALL SOT FUNCTIONS?}

Elucidation of all SOT functions in A. thaliana as a model plant is difficult for several reasons. Until now, ten out of 22 identified putative $A$. thaliana SOTs have been enzymatically characterized in vitro. The identified substrates were peptides, flavonoids, brassinosteroids, GIs, hydroxyjasmonate, and salicylic acid. As discussed before, prospects of phylogenetic analyses are very limited for SOTs. Already small changes in the sequence can lead to wide variations in substrate specificity. Even reliable predictions of yet uncharacterized SOTs in the organism A. thaliana are not possible. Therefore, reliable function prediction of SOTs in other plant species on the basis of $A$. thaliana sequences seems very unlikely.

Even when comparing SOTs that use the same class of substrates from different plant species, not only differences in kinetic values, but also variation of specificity toward different substrates and specific hydroxyl groups are noticed. For example, flavonol SOTs (AtSOT5, AtSOT8, AtSOT12, AtSOT13) from A. thaliana prefer kaempferol or flavonol glycosides as substrate and sulfate the hydroxyl groups at 3- and 7-position (Hashiguchi et al., 2013, 2014). But flavonol SOTs from F. chloraefolia and F. bidentis (L.) Kuntze prefer quercetin as substrates and sulfate at $3^{\prime}$ - and $4^{\prime}$-position (Varin et al., 1992, 1997b). Furthermore, all so far characterized $A$. thaliana SOTs sulfate a broad range of substrates. Most are functionally and biochemically related, but for example in case of AtSOT12, substrates with a wide range of biological functions are accepted as substrates.

Another difficulty is that SOTs are part of secondary metabolism and therefore fulfill species-specific functions. Hence, it is unlikely that all types of SOTs occur in A. thaliana. This is supported by the Pfam database research described in chapter 3.1. In A. thaliana, only the TPST contains a Sulfotransfer_2 domain (PF03567). The remaining AtSOTs all contain a Sulfotransfer_1 domain (PF00685), while the Sulfotransfer_3(PF13469) and Gal-3-O_sulfotr domain (PF06990) are only present in algae. Arylsulfotran_2 domain (PF14269) is only found in a single Ricinus communis sequence. In addition, SOT homologues in different plant species differ in their number of paralogs. For example, there were nine homologues of AtSOT18 found in B. rapa (Zang et al., 2009), which could differ in their characteristics.

All in all, it remains an important future task to clarify the biological functions and characteristics of the remaining
A. thaliana SOTs, not only by in vitro enzymatic assays, but in consideration of mutation, expression and localization studies, as well as metabolomics. Findings could at least be partly transferred and give valuable hints about specific SOTs in other species. Since A. thaliana is the most studied plant, complete characterization of all AtSOTs could also give more information about the connection of primary and secondary metabolisms in plants in general.

\section{FUTURE CHALLENGES}

Plant SOT research still remains a biological field with many open questions, especially in comparison with mammalian SOTs. In the model organism A. thaliana, only ten out of 22 SOTs have been enzymatically characterized in vitro so far. In many of these cases, the in vivo function is not elucidated yet. Some compounds, which were found to be sulfated by SOTs in vitro, could not be detected in vivo, as it was the case for sulfated glucoside flavonoids, sulfated by AtSOT8 (Hashiguchi et al., 2014). Furthermore, the function of sulfation or the sulfated compound is often not completely understood. Hence, for a deeper understanding it is advisable to follow in vitro enzymatic characterization with mutation, expression and localization studies.

For the remaining twelve putative $A$. thaliana SOTs, disregarding the pseudogene AtSOT2, no accepted substrates have been identified so far. Due to the enormous number of putative substrates and the restricted reliability of phylogenetic analyses, complete functional elucidation of all AtSOTs is an ambitious goal. Hence, recombinant expression and offering randomly chosen substrates seems like looking for a needle in a haystack. A more promising approach could be to feed wild-type and mutant plants with ${ }^{35} \mathrm{~S}$, followed by mass spectrometry analysis. This can facilitate the identification of newly sulfated compounds in vivo. Next steps could be the isolation of these compounds, depending on its availability and chemical properties. If possible, the compounds could be bound to a column and used for affinity chromatography of total protein preparations. This would be a very systematic approach and was already partly used for the successful identification of TPST (Komori et al., 2009).

Especially in pharmaceutical research, in silico analysis has become a powerful tool (Song et al., 2009). With the help of three dimensional structures of the target molecules, computational drug design becomes more and more promising. The three dimensional structure of one A. thaliana SOT (AtSOT12) has already been solved, but without including substrates into the crystals (Smith et al., 2004). A deeper understanding could be reached with the help of more solved structures with and without substrates and with additional knowledge about the enzymatic mechanism. Definitely identified binding sites, combined with protein modeling could give more specific hints about putative substrates of SOTs.

Another interesting field would be the elucidation of SOTs from more plant species, especially highly specialized ones. In order to cope with additional stress, plants growing in challenging environments often biosynthesize specific compounds. Its properties are often promising from a biological point of view, for a better understanding of stress response, but also interesting for medical or biotechnological applications. Zosteric acid [ $p$-(sulfo-oxy) cinnamic acid] from the seagrass Zostera 
marina, for example, has anti-fouling properties (Newby et al., 2006). In a patent, SOT involvement in biosynthesis was suggested, but not proven yet (Alexandratos, 1999). Additionally, in Zostera, Halophila, and Thalassia seagrass, the existence of sulfated flavones was indicated (Harborne and Williams, 1976). Furthermore sulfated polysaccharides were detected in seagrass (Aquino et al., 2005, 2011), freshwater plants (DantasSantos et al., 2012) and algae (Ngo and Kim, 2013). While the sulfation of polysaccharides is well-studied in humans (Kusche-Gullberg and Kjellén, 2003), no polysaccharide SOTs have been studied in plants yet. It is hypothesized that sulfated polysaccharides modify the cell wall in halophytes in order to increase salt tolerance (Aquino et al., 2005). They are also interesting for human nutrition and pharmaceutical products, because of their antioxidant, anti-allergic, anti-human immunodeficiency virus, anti-cancer and anticoagulant properties (Ngo and Kim, 2013).

Overall, substrate specificities, regulations, and catalytic mechanisms of plant SOTs are still poorly understood. Considering the large number of possible functions, further research on these enzymes remains a challenging field.

\section{ACKNOWLEDGMENTS}

We acknowledge support by Deutsche Forschungsgemeinschaft and Open Access Publishing Fund of Leibniz Universität Hannover. Experimental work in our laboratory is funded by the Deutsche Forschungsgemeinschaft (PA 764/10-1).

\section{REFERENCES}

Alexandratos, S. D. (1999). Synthesis and purification of zosteric acid. US Patent 5990336.

Allali-Hassani, A., Pan, P. W., Dombrovski, L., Najmanovich, R., Tempel, W., Dong, A., et al. (2007). Structural and chemical profiling of the human cytosolic sulfotransferases. PLoS Biol. 5:e97. doi: 10.1371/journal.pbio.0050097

Amano, Y., Tsubouchi, H., Shinohara, H., Ogawa, M., and Matsubayashi, Y. (2007). Tyrosine-sulfated glycopeptide involved in cellular proliferation and expansion in Arabidopsis. Proc. Natl. Acad. Sci. U.S.A. 104, 18333-18338. doi: 10.1073/pnas.0706403104

Aquino, R. S., Grativol, C., Mourão, P. S., and Meyer, P. (2011). Rising from the sea: correlations between sulfated polysaccharides and salinity in plants. PLoS ONE 6:e18862. doi: 10.1371/journal.pone.0018862

Aquino, R. S., Landeira-Fernandez, A. M., Valente, A. P., Andrade, L. R., and Mourão, P. A. (2005). Occurrence of sulfated galactans in marine angiosperms: evolutionary implications. Glycobiology 15, 11-20. doi: 10.1093/glycob/cwh138

Baek, D., Pathange, P., Chung, J.-S., Jiang, J., Gao, L., Oikawa, A., et al. (2010). A stress-inducible sulphotransferase sulphonates salicylic acid and confers pathogen resistance in Arabidopsis. Plant Cell Environ. 33, 1383-1392. doi: 10.1111/j.13653040.2010.02156.x

Berger, I., Guttman, C., Amar, D., Zarivach, R., and Aharoni, A. (2011). The molecular basis for the broad substrate specificity of human sulfotransferase 1A1. PLoS ONE 6:e26794. doi: 10.1371/journal.pone.0026794

Bidwell, L. M., Mcmanus, M. E., Gaedigk, A., Kakuta, Y., Negishi, M., Pedersen, L., et al. (1999). Crystal structure of human catecholamine sulfotransferase. J. Mol. Biol. 293, 521-530. doi: 10.1006/jmbi.1999.3153

Brown, P. D., Tokuhisa, J. G., Reichelt, M., and Gershenzon, J. (2003). Variation of glucosinolate accumulation among different organs and developmental stages of Arabidopsis thaliana. Phytochemistry 62, 471-481. doi: 10.1016/S00319422(02)00549-6

Celenza J. L., Quiel, J. A., Smolen, G. A., Merrikh, H., Silvestro, A. R., Normanly, J., etal. (2005). The Arabidopsis ATR1 Myb transcription factor controls indolic glucosinolate homeostasis. Plant Physiol. 137, 253-262. doi: 10.1104/pp.104.054395
Chen, R., Jiang, Y., Dong, J., Zhang, X., Xiao, H., Xu, Z., et al. (2012). Genome-wide analysis and environmental response profiling of SOT family genes in rice (Oryza sativa). Genes Genom. 34, 549-560. doi: 10.1007/s13258-012-0053-5

Clarke, D. B. (2010). Glucosinolates, structures and analysis in food. Anal. Methods 2, 310-325. doi: 10.1039/B9AY00280D

Cook, I., Wang, T., Falany, C. N., and Leyh, T. S. (2012). A nucleotide-gated molecular pore selects sulfotransferase substrates. Biochemistry 51, 5674-5683. doi: 10.1021/bi300631g

Cook, I., Wang, T., Falany, C. N., and Leyh, T. S. (2013). High accuracy in silico sulfotransferase models. J. Biol. Chem. 288, 34494-34501. doi: 10.1074/jbc.M113.510974

Dajani, R., Cleasby, A., Neu, M., Wonacott, A. J., Jhoti, H., Hood, A. M., et al. (1999). X-ray crystal structure of human dopamine sulfotransferase, SULT1A3: molecular modeling and quantitative structure-activity relationship analysis demonstrate a molecular basis for sulfotransferase substrate specificity. J. Biol. Chem. 274, 37862-37868. doi: 10.1074/jbc.274.53.37862

Dantas-Santos, N., Gomes, D. L., Costa, L. S., Cordeiro, S. L., Costa, M. S. S. P., Trindade, E. S., et al. (2012). Freshwater plants synthesize sulfated polysaccharides: heterogalactans from water hyacinth (Eicchornia crassipes). Int. J. Mol. Sci. 13, 961-976. doi: 10.3390/ijms13010961

Estavillo, G. M., Crisp, P. A., Pomsiriwong, W., Wirtz, M., Collinge, D., Carrie, C., et al. (2011). Evidence for a SAL1-PAP chloroplast retrograde pathway that functions in drought and high light signaling in Arabidopsis. Plant Cell 23, 39924012. doi: 10.1105/tpc.111.091033

Faulkner, I. J., and Rubery, P. H. (1992). Flavonoids and flavonoid sulphates as probes of auxin-transport regulation in Cucurbita pepo hypocotyl segments and vesicles. Planta 186, 618-625. doi: 10.1007/ BF00198044

Fenwick, G. R., and Heaney, R. K. (1983). Glucosinolates and their breakdown products in cruciferous crops, foods and feedingstuffs. Food Chem. 11, 249-271. doi: 10.1016/0308-8146(83)90074-90072

Finn, R. D., Bateman, A., Clements, J., Coggill, P., Eberhardt, R. Y., Eddy, S. R., et al. (2014). Pfam: the protein families database. Nucl. Acids Res. 42, D222-D230. doi: $10.1093 / \mathrm{nar} / \mathrm{gkt} 1223$

Frerigmann, H., and Gigolashvili, T. (2014). MYB34, MYB51, and MYB122 distinctly regulate indolic glucosinolate biosynthesis in Arabidopsis thaliana. Mol. Plant 7, 814-828. doi: 10.1093/mp/ssu004

Fukuta, M., Kobayashi, Y., and Uchimura, K. (1998). Molecular cloning and expression of human chondroitin 6-sulfotransferase. Biochim. Biophys. Acta 1399, 57-61.

Gamage, N., Barnett, A., Hempel, N., Duggleby, R. G., Windmill, K. F., Martin, J. L., et al. (2006). Human sulfotransferases and their role in chemical metabolism. Toxicol. Sci. 90, 5-22. doi: 10.1093/toxsci/kfj061

Gidda, S. K., Miersch, O., Levitin, A., Schmidt, J., Wasternack, C., and Varin, L. (2003). Biochemical and molecular characterization of a hydroxyjasmonate sulfotransferase from Arabidopsis thaliana. J. Biol. Chem. 278, 17895-17900. doi: 10.1074/jbc.M211943200

Gidda, S. K., and Varin, L. (2006). Biochemical and molecular characterization og flavonoid 7-sulfotransferase from Arabidopsis thaliana. Plant Physiol. Biochem. 44, 628-636 doi: 10.1074/jbc.M211943200

Gigolashvili, T., Berger, B., Mock, H. -P., Muller, C., Weisshaar, B., and Flügge, U.-I. (2007a). The transcription factor HIG1/MYB51 regulates indolic glucosinolate biosynthesis in Arabidopsis thaliana. Plant J. 50, 886-901. doi: 10.1111/j.1365313X.2007.03099.x

Gigolashvili, T., Yatusevich, R., Berger, B., Müller, C., and Flügge, U.-I. (2007b). The R2R3-MYB transcription factor HAG1/MYB28 is a regulator of methioninederived glucosinolate biosynthesis in Arabidopsis thaliana. Plant J. 51, 247-261. doi: 10.1111/j.1365-313X.2007.03133.x

Gigolashvili, T., Engqvist, M., Yatusevich, R., Müller, C., and Flügge, U.-I. (2008). HAG2/MYB76 and HAG3/MYB29 exert a specific and coordinated control on the regulation of aliphatic glucosinolate biosynthesis in Arabidopsis thaliana. New Phytol. 177, 627-642. doi: 10.1111/j.1469-8137.2007. 02295.x

Gigolashvili, T., Geier, M., Ashykhmina, N., Frerigmann, H., Wulfert, S., Krueger, S., et al. (2012). The Arabidopsis thylakoid ADP/ATP carrier TAAC has an additional role in supplying plastidic phosphoadenosine 5-phosphosulfate to the cytosol. Plant Cell 24, 4187-4204. doi: 10.1105/tpc.112.101964

Glendening, T. M., and Poulton, J. E. (1990). Partial purification and characterization of a $3^{\prime}$-phosphoadenosine $5^{\prime}$-phosphosulfate: desulfoglucosinolate 
sulfotransferase from cress (Lepidium sativum). Plant Physiol. 94, 811-818. doi: 10.1104/pp.94.2.811

Graser, G., Oldham, N. J., Brown, P. D., Temp, U., and Gershenzon, J. (2001). The biosynthesis of benzoic acid glucosinolate esters in Arabidopsis thaliana. Phytochemistry 57, 23-32. doi: 10.1016/S0031-9422(00)00501-X

Halkier, B. A. (1999). “Glucosinolates,” in Naturally Occurring Glycosides: Chemistry, Distribution and Biological Properties, ed. R. Ikan (New York: John Wiley \& Sons Ltd), 193-223.

Hanai, H., Nakayama, D., Yang, H., Matsubayashi, Y., Hirota, Y., and Sakagami, Y. (2000). Existence of a plant tyrosylprotein sulfotransferase: novel plant enzyme catalyzing tyrosine O-sulfation of preprophytosulfokine variants in vitro. FEBS Lett. 470, 97-101. doi: 10.1016/S0014-5793(00)01299-0

Harborne, J. B., and Williams, C. A. (1976). Occurrence of sulphated flavones and caffeic acid esters in members of the fluviales. Biochem. Syst. Ecol. 4, 37-41. doi 10.1016/0305-1978(76)90007-7

Hashiguchi, T., Sakakibara, Y., Hara, Y., Shimohira, T., Kurogi, K., Akashi, R., et al. (2013). Identification and characterization of a novel kaempferol sulfotransferase from Arabidopsis thaliana. Biochem. Biophys. Res. Commun. 434, 829-835. doi: 10.1016/j.bbrc.2013.04.022

Hashiguchi, T., Sakakibara, Y., Shimohira, T., Kurogi, K., Yamasaki, M., Nishiyama, K., et al. (2014). Identification of a novel flavonoid glycoside sulfotransferase in Arabidopsis thaliana. J. Biochem. 155, 91-97. doi: 10.1093/jb/mvt102

Hernàndez-Sebastiá, C., Varin, L., and Marsolais, F. (2008). "Sulfotransferases from plants, algae and phototrophic bacteria," in Sulfur Metabolism in Phototrophic Organisms, eds R. Hell, C. Dahl, D. Knaff, and T. Leustek (Amsterdam: Springer), 111-130.

Hirai, M. Y., Klein, M., Fujikawa, Y., Yano M., Goodenowe, D. B., Yamazaki, Y., et al. (2005). Elucidation of gene-to-gene networks in Arabidopsis by integration of metabolomics and transcriptomics. J. Biol. Chem. 280, 25590-25595. doi: 10.1074/jbc.M502332200

Hirai, M. Y., Sugiyama, K., Sawada, Y., Tohge, T., Obayashi, T., Suzuki, A., et al (2007). Omics-based identification of Arabidopsis Myb transcription factors regulating aliphatic glucosinolate biosynthesis. Proc. Natl. Acad. Sci. U.S.A. 104, 6478-6483. doi: 10.1073/pnas.0611629104

Huseby, S., Koprivova, A., Lee, B.-R., Saha, S., Mithen, R., Wold, A.-B., et al. (2013). Diurnal and light regulation of sulphur assimilation and glucosinolate biosynthesis in Arabidopsis. J. Exp. Bot. 64, 1039-1048. doi: 10.1093/jxb/ ers378

Jensen, L. M., Halkier, B. A., and Burow, M. (2014). How to discover a metabolic pathway? An update on gene identification in aliphatic glucosinolate biosynthesis, regulation and transport. Biol. Chem. 395, 529-543. doi: 10.1515/hsz-2013-0286

Kakuta, Y., Pedersen, L. G., Carter, C. W., Negishi, M., and Pedersen, L. C. (1997). Crystal structure of estrogen sulphotransferase. Nature Struct. Biol. 4, 904-908. doi: 10.1038/nsb1197-904

Kakuta, Y., Petrotchenko, E. V., Pedersen, L. C., and Negishi, M. (1998) The sulfuryl transfer mechanism. J. Biol. Chem. 273, 27325-27330. doi 10.1074/jbc.273.42.27325

Klaassen, C. D., and Boles, J. W. (1997). The importance of $3^{\prime}$-phosphoadenosine $5^{\prime}$-phosphosulfate (PAPS) in the regulation of sulfation. FASEB J. 11, 404-418.

Klein, M., and Papenbrock, J. (2004). The multi-protein family of Arabidopsis sulphotransferases and their relatives in other plant species. J. Exp. Bot. 55, 1809-1820. doi: 10.1093/jxb/erh183

Klein, M., and Papenbrock, J. (2008). "Sulfotransferases and their tole in glucosinolate biosynthesis," in Sulfur Assimilation and Abiotic Stress in Plants, eds. N. Khan, S. Singh, and S. Umar (Berlin: Springer), 149-166. doi: 10.1007/978-3-540-7 6326-0_7

Klein, M., and Papenbrock, J. (2009). Kinetic parameters of desulfoglucosinolate sulfotransferases. Physiol. Plant. 135, 140-149. doi: 10.1111/j.13993054.2008.01182.x

Klein, M., Reichelt, M., Gershenzon, J., and Papenbrock, J. (2006). The three desulfo-glucosinolate sulfotransferase proteins in Arabidopsis have different substrate specificities and are differentially expressed. FEBS J. 273, 122-136. doi: 10.1111/j.1742-4658.2005.05048.x

Kliebenstein, D. J., Kroymann, J., Brown, P., Figuth, A., Pedersen, D., Gershenzon, J., et al. (2001a). Genetic control of natural variation in Arabidopsis glucosinolate accumulation. Plant Physiol. 126, 811-825. doi: 10.1104/pp.126.2.811

Kliebenstein, D. J., Lambrix, V. M., Reichelt, M., and Gershenzon, J. (2001b). Gene duplication in the diversification of secondary metabolism: Tandem 2-oxoglutarate-dependent dioxygenases control glucosinolate biosynthesis in Arabidopsis. Plant Cell 13, 681-693. doi: 10.1105/tpc.13.3.681

Komori, R., Amano, Y., Ogawa-Ohnishi, M., and Matsubayashi, Y. (2009). Identification of tyrosylprotein sulfotransferase in Arabidopsis. Proc. Natl. Acad. Sci. U.S.A. 106, 15067-15072. doi: 10.1073/pnas.0902801106

Kusche-Gullberg, M., and Kjellén, L. (2003). Sulfotransferases in glycosaminoglycan biosynthesis. Curr. Opin. Struct. Biol. 13, 605-611. doi: 10.1016/j.sbi.2003.08.002

Labonne, J. J. D., Goultiaeva, A., and Shore, J. S. (2009). High-resolution mapping of the S-locus in Turnera leads to the discovery of three genes tightly associated with the S-alleles. Mol. Genet. Genomics 281, 673-685. doi: 10.1007/s00438-0090439-5

Lacomme, C., and Roby, D. (1996). Molecular cloning of a sulfotransferase in Arabidopsis thaliana and regulation during development and in response to infection with pathogenic bacteria. Plant Mol. Biol. 30, 995-1008. doi: 10.1007/ BF00020810

Lee, B. R., Huseby, S., Koprivova, A., Chételat, A., Wirtz, M., Mugford, S. T., et al. (2012). Effects of fou8/fryl mutation on sulfur metabolism: is decreased internal sulfate the trigger of sulfate starvation response? PLoS ONE 7:e39425. doi: 10.1371/journal.pone.0039425

Lewis, D. R., Ramirez, M. V., Miller, N. D., Vallabhaneni, P., Ray, W. K., Helm, R. F., et al. (2011). Auxin and ethylene induce flavonol accumulation through distinct transcriptional networks. Plant Physiol. 156, 144-164. doi: $10.1104 / \mathrm{pp} .111 .172502$

Li, Y., Sawada, Y., Hirai, A., Sato, M., Kuwahara, A., Yan, X., et al. (2013). Novel insights into the function of Arabidopsis R2R3-MYB transcription factors regulating aliphatic glucosinolate biosynthesis. Plant Cell Physiol. 54, 1335-1344. doi: $10.1093 / \mathrm{pcp} / \mathrm{pct} 085$

Luczak, S., Forlani, F., and Papenbrock, J. (2013). Desulfo-glucosinolate sulfotransferases isolated from several Arabidopsis thaliana ecotypes differ in their sequence and enzyme activities. Plant Physiol. Biochem. 63, 15-23. doi: 10.1016/j.plaphy.2012.11.005

Malitsky, S., Blum, E., Less, H., Venger, I., Elbaz, M., Morin, S., et al. (2008). The transcript and metabolite networks affected by the two clades of Arabidopsis glucosinolate biosynthesis regulators. Plant Physiol. 148, 2021-2049. doi: 10.1104/pp.108.124784

Marsolais, F., Boyd, J., Paredes, Y., Schinas, A.-M., Garcia, M., Elzein, S., et al. (2007). Molecular and biochemical characterization of two brassinosteroid sulfotransferases from Arabidopsis, AtST4a (At2g14920) and AtST1 (At2g03760). Planta 225, 1233-1244. doi: 10.1007/s00425-006-0413-y

Marsolais, F., Gidda, S. K., Boyd, J., and Varin, L. (2000). Structural and functional similarity with mammalian enzymes. Rec. Adv. Phytochem. 34, 433-456. doi: 10.1371/journal.pone.0104594

Marsolais, F., Sebastià, C. H., Rousseau, A., and Varin, L. (2004). Molecular and biochemical characterization of BNST4, an ethanol-inducible steroid sulfotransferase from Brassica napus, and regulation of BNST genes by chemical stress and during development. Plant Sci. 166, 1359-1370. doi: 10.1016/j.plantsci.2004.01.019

Marsolais, F., and Varin, L. (1995). Identification of amino acid residues critical for catalysis and cosubstrate binding in the flavonol 3-sulfotransferase. J. Biol. Chem. 270, 30458-30463. doi: 10.1074/jbc.270.51.30458

Matsubayashi, Y., and Sakagami, Y. (1996). Phytosulfokine, sulfated peptides that induce the proliferation of single mesophyll cells of Asparagus officinalis L. Proc. Natl. Acad. Sci. U.S.A. 93, 7623-7627. doi: 10.1073/pnas.93.15.7623

Matsuzaki, Y., Ogawa-Ohnishi, M., Mori, A., and Matsubayashi, Y. (2010). Secreted peptide signals required for maintenance of root stem cell niche in Arabidopsis. Science 329, 1065-1067. doi: 10.1126/science.1191132

Mithen, R. (2001). Glucosinolates - biochemistry, genetics and biological activity. Plant Growth Regul. 34, 91-103. doi: 10.1023/A:1013330819778

Møldrup, M. E., Geu-Flores, F., Olsen, C. E., and Halkier, B. A. (2011). Modulation of sulfur metabolism enables efficient glucosinolate engineering. BMC Biotechnol. 11:12. doi: 10.1186/1472-6750-11-12.

Mugford, S. G., Yoshimoto, N., Reichelt, M., Wirtz, M., Hill, L., Mugford, S. T., et al. (2009). Disruption of adenosine-5' -phosphosulfate kinase in Arabidopsis reduces levels of sulfated secondary metabolites. Plant Cell 21, 910-927. doi: 10.1105/tpc.109.065581

Newby, B.-M. Z., Cutright, T., Barrios, C., and Xu, Q. (2006). Zosteric acid-An effective antifoulant for reducing fresh water bacterial attachment on coatings. JCT Res. 3, 69-76. doi: 10.1007/s11998-006-0007-4 
Ngo, D.-H., and Kim, S.-K. (2013). Sulfated polysaccharides as bioactive agents from marine algae. Int. J. Biol. Macromol. 62, 70-75. doi: 10.1016/j.ijbiomac. 2013.08.036

Niehrs, C., Beisswanger, R., and Huttner, W. B. (1994). Protein tyrosine sulfation, 1993 - an update. Chem. Biol. Interact. 92, 257-271. doi: 10.1016/00092797(94)90068-X

Pi, N., Hoang, M. B., Gao, H., Mougous, J. D., Bertozzi, C. R., and Leary, J. A. (2005). Kinetic measurements and mechanism determination of Stf0 sulfotransferase using mass spectrometry. Anal. Biochem. 341, 94-104. doi: 10.1016/j.ab.2005.02.004

Pi, N., Yu, Y., Mougous, J. D., and Leary, J. A. (2004). Observation of a hybrid random ping-pong mechanism of catalysis for NodST: a mass spectrometry approach. Protein Sci. 13, 903-912. doi: 10.1110/ps.03581904

Piotrowski, M., Schemenewitz, A., Lopukhina, A., Muller, A., Janowitz, T., Weiler, E. W., et al. (2004). Desulfoglucosinolate sulfotransferases from Arabidopsis thaliana catalyze the final step in the biosynthesis of the glucosinolate core structure. $J$. Biol. Chem. 279, 50717-50725. doi: 10.1074/jbc.M407681200

Rausch, T., and Wachter, A. (2005). Sulfur metabolism: a versatile platform for launching defence operations. Trends Plant Sci. 10, 503-509. doi 10.1016/j.tplants.2005.08.006

Rivoal, J., and Hanson, A. D. (1994). Choline-O-sulfate biosynthesis in plants (Identification and partial characterization of a salinity-inducible choline sulfotransferase from species of Limonium (Plumbaginaceae). Plant Physiol. 106 1187-1193

Rouleau, M., Marsolias, F., Richard, M., Nicolle, L., Voigt, B., Adam, G., et al (1999). Inactivation of brassinosteroid biological activity by a salicylate-inducible steroid sulfotransferase from Brassica napus. J. Biol. Chem. 274, 20925-20930. doi 10.1074/jbc.274.30.20925

Sandhu, K. S., and Neff, M. M. (2013). The Arabidopsis gene ATST4a in not a typical brassinosteroid catabolic gene. Plant Signal. Behav. 8, 10. doi: 10.4161/psb.26847

Shimojima, M., Hoffmann-Benning, S., Garavito, R. M., and Benning, C. (2005). Ferredoxin-dependent glutamate synthase moonlights in plant sulfolipid biosynthesis by forming a complex with SQD1. Arch. Biochem. Biophys. 436, 206-214. doi: 10.1016/j.abb.2005.02.005

Smith, D. W., Johnson, K. A., Bingman, C. A., Aceti, D. J., Blommel, P. G., Wrobel, R. L., et al. (2004). Crystal structure of At2g03760, a putative steroid sulfotransferase from Arabidopsis thaliana. Proteins 57, 854-857. doi: 10.1002/prot.20258

Sønderby, I. E., Burow, M., Rowe, H. C., Kliebenstein, D. J., and Halkier, B. A. (2010). A complex interplay of three R2R3 MYB transcription factors determines the profile of aliphatic glucosinolates in Arabidopsis. Plant Physiol. 153, 348-363. doi: 10.1104/pp.109.149286

Sønderby, I. E., Hansen, B. G., Bjarnholt, N., Ticconi, C., Halkier, B. A., and Kliebenstein, D. J. (2007). A system biology approach identifies a R2R3 MYB gene subfamily with distinct and overlapping functions in regulation of aliphatic glucosinolates. PLoS ONE 2:e1322. doi: 10.1371/journal.pone.0001322

Song, C. M., Lim, S. J., and Tong, J. C. (2009). Recent advances in computer-aided drug design. Brief. Bioinf. 10, 579-591. doi: 10.1093/bib/bbp023

Vakiani, E., Luz, J. G., and Buck, J. (1998). Substrate specificity and kinetic mechanism of the insect sulfotransferase, retinol dehydratase. J. Biol. Chem. 273, 35381-35387. doi: 10.1074/jbc.273.52.35381.

Varin, L., Chamberland, H., Lafontaine, J. G., and Richard, M. (1997a). The enzyme involved in sulfation of the turgorin, gallic acid 4-O-(beta-D-glucopyranosyl$6^{\prime}$-sulfate) is pulvini-localized in Mimosa pudica. Plant J. 12, 831-837. doi: 10.1046/j.1365-313X.1997.12040831.x

Varin, L., Marsolais, F., Richard, M., and Rouleau, M. (1997b). Biochemistry and molecular sulfotransferases biology of plant sulfotransferases. FASEB J. 11, 517-525.
Varin, L., Deluca, V., Ibrahim, R. K., and Brisson, N. (1992). Molecular characterization of two plant flavonol sulfotransferases. Proc. Natl. Acad. Sci. U.S.A. 89, 1286-1290. doi: 10.1073/pnas.89.4.1286

Varin, L., and Ibrahim, R. K. (1992). Novel flavonol 3-sulfotransferase. Purification, kinetic properties, and partial amino acid sequence. J. Biol. Chem. 267, 18581863.

Varin, L., and Spertini, D. (2003). Desulfoglucosinolate sulfotransferases, sequences coding the same and uses thereof for modulating glucosinolate biosynthesis in plants. Patent WO 2003010318.

Wang, H., Wu, J., Sun, S., Liu, B., Cheng, F., Sun, R., et al. (2011). Glucosinolate biosynthetic genes in Brassica rapa. Gene 487, 135-142. doi: 10.1016/j.gene.2011.07.021

Wang, T., Cook, I., Falany, C. N., and Leyh, T. S. (2014). Paradigms of sulfotransferase catalysis - the mechanism of SULT2A1. J. Biol. Chem. 289, 26474-2680. doi: 10.1074/jbc.M114.573501

Weinshilboum, R. M., and Otterness, D. M. (1994). "Sulfotransferase enzymes," in Handbook of Experimental Pharmacology, ed. Kauffman (Berlin: Springer-Verlag), $45-78$.

Wittstock, U., and Halkier, B. A. (2002). Glucosinolate research in the Arabidopsis era. Trends Plant Sci. 7, 263-270. doi: 10.1016/S1360-1385(02) 02273-2

Yamashino, T., Kitayama, M., and Mizuno, T. (2013). Transcription of ST2A encoding a sulfotransferase family protein that is involved in jasmonic acid metabolism is controlled according to the circadian clockand PIF4/PIF5-mediated external coincidence mechanism in Arabidopsis thaliana. Biosci. Biotechnol. Biochem. 77, 2454-2460. doi: 10.1271/ bbb. 130559

Zang, Y.-X., Kim, H. U., Kim, J. A., Lim, M.-H., Jin, M., Lee, S. C., et al. (2009). Genome-wide identification of glucosinolate synthesis genes in Brassica rapa. FEBS J. 276, 3559-3574. doi: 10.1111/j.1742-4658.2009. 07076.x

Zhai, G., Lehmler, H.-J., and Schnoor, J. L. (2013). Sulfate metabolites of 4monochlorobiphenyl in whole poplar plants. Environ. Sci. Technol. 47, 557-562. doi: 10.1021/es303807f

Zhang, H., Varmalova, O., Vargas, F. M., Falany, C. N., and Leyh, T. S. (1998). Sulfuryl transfer: the catalytic mechanism of human estrogen sulfotransferase. J. Biol. Chem. 273, 10888-10892.doi: 10.1074/jbc.273. 18.10888

Conflict of Interest Statement: The authors declare that the research was conducted in the absence of any commercial or financial relationships that could be construed as a potential conflict of interest.

Received: 11 August 2014; accepted: 28 September 2014; published online: 16 October 2014.

Citation: Hirschmann F, Krause F and PapenbrockJ (2014) The multi-protein family of sulfotransferases in plants: composition, occurrence, substrate specificity, and functions. Front. Plant Sci. 5:556. doi: 10.3389/fpls.2014.00556

This article was submitted to Plant Physiology, a section of the journal Frontiers in Plant Science.

Copyright (C) 2014 Hirschmann, Krause and Papenbrock. This is an open-access article distributed under the terms of the Creative Commons Attribution License (CC BY). The use, distribution or reproduction in other forums is permitted, provided the original author(s) or licensor are credited and that the original publication in this journal is cited, in accordance with accepted academic practice. No use, distribution or reproduction is permitted which does not comply with these terms. 